\title{
Biased collective memories and historical overclaiming: An availability heuristic account
}

\author{
Jeremy K. Yamashiro ${ }^{1,2}$ (D) $\cdot$ Henry L. Roediger III $^{3}$ \\ Published online: 25 August 2020 \\ (C) The Psychonomic Society, Inc. 2020
}

\begin{abstract}
People tend to overclaim historical influence for their own ingroup, in a phenomenon called ingroup inflation. Although this overclaiming has been empirically demonstrated in the USA and other nations, the cognitive mechanisms underlying it have been largely conjectural. We test one such proposed mechanism: the application of the availability heuristic to a biased collective memory. Collective memories in the psychological sense are shared memories held individually by members of a group that pertain to their group identity. Using measures of retrieval fluency, we show that asymmetrical accessibility for collective memories favoring ingroup - versus outgroup - relevant historical events is correlated with overclaiming, and that reducing this asymmetry through targeted retrieval of outgroup-relevant events reduces overclaiming (Experiments 1 and 2). We also suggest that ingroup inflation arises because of retrieval fluency per se, rather than more stable asymmetries in knowledge or eventspecific judgments of importance (Experiment 3). Together, these studies suggest some cognitive bases of collective overclaiming and cognitive interventions that might attenuate these biased judgments.
\end{abstract}

Keywords Collective memory $\cdot$ Availability heuristic $\cdot$ Memory $\cdot$ Collective overclaiming $\cdot$ Ingroup inflation

People frequently represent their history through a chauvinistic lens (Roediger et al., 2020). While scholars in the humanistic disciplines of memory studies have documented and theorized this tendency extensively, empirically oriented psychologists have also recently begun inquiring into the role of cognitive bias in lay representations of history (Hirst et al., 2018; Roediger \& Abel, 2015). One developing line of research examines ingroup inflation, a bias in

Memory, like war, is often asymmetrical.

-Viet Thanh Nguyen (2016), Nothing Ever Dies

Electronic supplementary material The online version of this article (https://doi.org/10.3758/s13421-020-01090-w) contains supplementary material, which is available to authorized users.

Jeremy K. Yamashiro

yamashiro@ucsc.edu

1 Princeton School for Public and International Affairs, Princeton University, Princeton, NJ, USA

2 Department of Psychology, University of California, Santa Cruz, Social Sciences 2, Room 365, 1156 High Street, Santa Cruz, CA 95064, USA

3 Department of Psychological and Brain Sciences, Washington University in St. Louis, St. Louis, MO, USA judgment in which people reliably attribute more historical influence to their own group than non-group members do (Churchill et al., 2019). Both affective and cognitive factors likely contribute to this collective overclaiming (Putnam et al., 2018). In the current set of studies, we examine one cognitive mechanism conjectured to underly ingroup inflation: the availability heuristic, ${ }^{1}$ or the rule of thumb by which people make probability estimations based on the ease with which instances of an event come to mind (Tversky \& Kahneman, 1973). Precisely estimating various groups' contributions to history is a difficult task; people may substitute for it the simpler task of judging how easily specific historical events come to mind for specific groups. If people apply the availability heuristic while making judgments about historical influence, inflated estimates of the ingroup's historical influence should scale with the relative asymmetry in accessibility between ingroup- and outgroup-related events in memory. The

\footnotetext{
${ }^{1}$ Tulving and Pearlstone (1966) differentiated accessibility - the ease with which a previously encoded memory may be retrieved, from availability whether or not a memory has been encoded and stored. Unfortunately, Tversky and Kahneman's sense of availability correspondes not to availability in Tulving and Pearlstone's framework, but to accessibility. For current purposes, we will use both terms - "availability" and "accessibility" - to mean the ease with which a previously encoded memory may be retrieved, as is common practice in the literature on the availability heuristic.
} 
availability heuristic account of ingroup inflation has received some preliminary support in studies using laboratory materials and fictitious countries (Ross et al., 2020), but the current studies are the first to demonstrate the relation between retrieval bias and chauvinistic judgments of historical influence using collective memories of real-world social groups. Having gained some insight into cognitive factors underlying ingroup inflation, we suggest methods to deflate this overclaiming and support social identities less characterized by chauvinistic bias.

\section{Ingroup inflation}

Recent empirical work has developed methods to measure and precisely quantify the general tendency to overclaim historical influence for one's ingroup. For instance, when asked how much people from each state contributed to overall US history, Americans tend to allocate a larger proportion of history to people from their home state than do other Americans who are not from that state, for example, Virginians claim that a higher proportion of American history happened in Virginia than other Americans do (Putnam et al., 2018). Such overclaiming can also appear at the level of nationality. Citizens of 35 countries claimed for themselves extraordinarily high proportions of credit for world history (Zaromb et al., 2018), and people from World War II combatant countries reliably claimed greater responsibility for victory or the war effort than they conceded to their country's allies, each seeming to proclaim that "we won the war - and no one else did much" (Roediger et al., 2019). Putnam et al. (2018) suggested two general classes of psychological mechanisms that could potentially explain why such overclaiming occurs: affective/motivational factors and cognitive factors.

\section{Affective/motivational factors}

In prior demonstrations of ingroup inflation, the researchers conjectured that some of this observed inflation could occur because people are motivated by ingroup affection, and, relatedly, that thinking of the ingroup as having been an influential actor in history is ego-protective (Putnam et al., 2018; Zaromb et al. 2018). This motivational mechanism has received some support, in that people who more strongly endorse the group-oriented moral values of loyalty, respect for authority, and sanctity (Graham et al., 2009), for whom a chauvinistic rendering of the past may be more emotionally satisfying, tend to show higher degrees of ingroup inflation (Churchill et al., 2019).

\section{Cognitive factors}

Although such affective/motivational factors undoubtedly play a role in explaining the effect, in the current studies we focus on cognitive factors underlying ingroup inflation. Ross and Sicoly's (1979) work on egocentric biases in availability and attribution provides a model. In one study, Ross and Sicoly showed that partners in a marriage each tended to overestimate the proportion of household chores they themselves had done and underestimated the proportion their partner had done. This led to the paradoxical conclusion that both partners believed themselves to have taken on most of the household chores. The overclaiming effect replicated in members of basketball teams and between graduate students and their advisers. Ross and Sicoly (1979) attributed these incongruent judgments to differences in availability. When making judgments about their respective contributions, people attempt to recall examples of their own activities, as well as examples of other people's activities. They retrieve their own activities more fluently and with greater phenomenological vividness, relative to the activities of others.

This asymmetry in the ease of recall then biases judgments of responsibility via the availability heuristic. This cognitive shortcut often leads to reasonably accurate judgments; events that come to mind easily often are in fact events that occur frequently. However, not all events that come to mind easily do so because they occur frequently. For example, people estimate that the likelihood of death by accident, by tornado, or by botulism is much higher than death by stroke, asthma, or diabetes, although these diseases kill orders of magnitude more frequently than do the more exotic causes (Lichtenstein et al., 1978). Events that are more concrete and emotionally vivid may be more accessible in memory for reasons that are orthogonal to their true likelihood. Additionally, as we shall suggest, events that are relevant to our social identities may also be more accessible than events that are not.

\section{Bias in collective memory}

We are thus concerned with the application of the availability heuristic to an asymmetrically accessible store of collective memories. We define collective memories psychologically, as the memories and historical knowledge held by individual members of a group that pertain to their group identity (Hirst \& Manier, 2008; Wertsch \& Roediger, 2008). Collective memory tends to recount the shared past from a group-centric, singular, committed perspective, centering the ingroup in history (Novick, 1999; Wertsch, 2002). To translate this into the cognitive phenomenon of ingroup inflation, ingroup-relevant events may be more accessible in memory than outgrouprelevant events. However, unlike married couples thinking of their respective chores, it is not immediately obvious that 
such a bias in the accessibility of collective memories arises from the same mechanisms as bias in episodic memory. One important distinction is that when it comes to collective memory as defined above, people likely did not experience firsthand the majority of group-identity-relevant events they can recall. For instance, although most Americans could tell the more or less collectively shared story of how the American colonies gained independence from Britain, no one alive personally experienced the Revolutionary War. This is also true for more recent events. Although a small number of living Americans may have witnessed the Pearl Harbor attacks or, for that matter, 9/11 - in person, most Americans learned about these events vicariously. Unlike availability biases in episodic memory, which are based on different personal experiences, there is less a priori reason for assuming that historical knowledge would show the analogous asymmetries in retrieval fluency that favor events relevant to ingroup social identity.

If the availability heuristic underlies ingroup inflation, three specific predictions should hold. First, there should be a parochial knowledge bias in collective memory. A parochial knowledge bias would be apparent if historical events involving the ingroup could be accessed more fluently than historical events involving outgroups. Again, as the majority of historical events - ingroup- or outgroup-relevant - were not experienced first-hand, this point cannot be taken for granted. Second, the degree of parochial bias, i.e., how much less accessible outgroup-relevant events are relative to ingroup events, should correlate positively with ingroup inflation, which reflects more abstract judgments about historical influence. Finally, if ingroup inflation occurs because people consult an asymmetrical base of collective memory while making judgments about historical influence, ingroup inflation should attenuate if we equate the relative accessibility of ingroup- and outgroup-relevant historical events.

\section{The current studies}

In the following studies, we first aimed to replicate the finding of ingroup inflation using a variation on Putnam et al.'s (2018) methodology for studying state narcissism, which is what they called ingroup inflation in the context of US states. Second, we attempted, for the first time, to demonstrate a parochial knowledge bias. We tested for the parochial knowledge bias using two methods, a retrieval fluency task for historical events (Experiment 1) and a recognition task using multiplechoice questions (Experiment 3). Prior literature has used relative retrieval fluency between two target domains as a measure of cognitive bias, most typically in calculating emotional biases in autobiographical memory and personal future thought (MacLeod et al., 1993; MacLeod \& Byrne, 1996; MacLeod et al., 1997) and in collective future thought
(Shrikanth et al., 2018). In these cases, researchers compare retrieval fluency for positive versus negative memories or imagined future events. We adapt this tool for measuring bias in the accessibility of ingroup- versus outgroup-relevant historical events, rather than positive or negative memories and future thoughts. In Experiments 1 and 3, we sought to demonstrate that the parochial knowledge bias correlates positively with ingroup inflation. In Experiment 2, we attempted to demonstrate that attenuating the parochial knowledge bias by increasing the relative availability of outgroup relevant events decreases ingroup inflation.

\section{Experiment 1}

\section{Methods}

\section{Participants}

We aimed to recruit a total of 100 participants from each of three US states - Massachusetts, Virginia, and California - for a total sample of 300. Massachusetts, Virginia, and California were selected because participants from those states had demonstrated high levels of ingroup inflation in prior research (Churchill et al., 2019; Putnam et al., 2018). A G*Power power analysis for an ingroup inflation effect similar to that reported in prior literature $(d=0.41)$, with alpha at .05 , power of 0.80 , and an allocation ratio of 2 would suggest that 168 participants ( 56 per state) would be necessary for sufficient power. The correlation between parochial knowledge bias and ingroup inflation, if it were at least as strong as the correlation between endorsement of binding values and ingroup inflation reported in Churchill et al. (2019) (i.e., $r=$ .17), would require 212 participants. G*Power Protocols for these two power analyses are provided in Appendix I of the Online Supplemental Materials (OSM). A total initial sample of 303 participants was recruited via Amazon's Mechanical Turk. Fourteen participants were excluded for providing nonsense answers during the fluency task, for a final sample of 289 participants $\left(\mathrm{N}_{M A}=96, \mathrm{~N}_{V A}=93, \mathrm{~N}_{C A}=100\right.$.). This final sample retained satisfactory power for the planned tests. Participants had to live in one of the three target states, and they must not have lived outside of their home state prior to the age of 18 years, or have lived outside of their home state for more than 4 years total. They were required to read and speak fluent English, although English did not need to be their first language.

Their mean age was 34 years, with a range from 18 to 79 . Forty-four percent of the sample identified as female. Regarding highest educational attainment, $0.03 \%$ had less than a high school degree, $7 \%$ had a high school degree or equivalent, 9\% had an Associate's degree, 19\% had some college, $49 \%$ had a Bachelors degree, $12 \%$ had a Masters 
degree, $3.5 \%$ had a professional degree or doctorate. When asked on a five-point scale the extent to which the US state in which they grew up constituted an important part of their personal identity, participants chose a mean of $2.37,95 \%$ CI [2.23, 2.51], between "A moderate amount" and "A lot." Participants from different states did not differ in the extent to which their home state was important to their personal identity, $p>.05$.

Participants from each of the three states were recruited via separate recruitment pages on MTurk, and all participants were directed to a single Qualtrics survey. They selected their home state from a set of choices: Virginia, Massachusetts, California, or Other. If they selected "Other" they were automatically disqualified. Passing this check, participants engaged in two tasks, a historical event fluency task and historical influence ratings, with task order randomized for each participant.

\section{Fluency task}

In the fluency task, participants saw the cue: "Many events important to US history occurred in [Target State]. Please list as many of them as you can as fast as you can. Separate each event by a comma." Participants saw [Target State] as the state they had selected as their home state at the beginning of the study: Massachusetts, Virginia, or California. Participants saw one state at a time, and the order in which states were presented was randomized for each participant. All participants listed events for all three states. Participants typed historical events into a dialogue box under the question. Each question was timed, and after 1 min participants were automatically advanced to the next page, which cued events for the next state, and so on until participants had provided events for all three target states. Participants were not able to advance to the next page until the minute had expired.

\section{Influence-rating task}

In the historical influence-rating task, participants estimated the proportion of US history that could be attributed to each of the three states. Participants were asked, "In terms of percentage, what was [Target State's] contribution to the history of the United States?" Participants moved a slider, initially set to 0 with a maximum value of 100 , to indicate how much of US history could be attributed to each state. There was one state rating per page, with the order in which the three states were presented randomized for each participant. This section was not timed, and participants could advance at their own pace.

Finally, participants provided the following demographic information: age, how long they had lived in their home state, how many years they had lived outside their home state, gender, ethnicity, what sort of area they grew up in (rural, small town, suburban, or urban), highest level of education, yearly household income, how many classes on the history of their home state they had taken, how important the state they grew up in was to their personal identity, how much being an American was important to their personal identity, political affiliation, and the political party for which they usually vote. The study took approximately $6.5 \mathrm{~min}$ to complete, and participants were reimbursed US $\$ 1$. The Washington University in St. Louis Human Research Protections Office exempted the study from IRB review. The full Qualtrics survey is provided in Appendix II of the OSM.

\section{Results}

\section{Ingroup inflation}

All bracketed numbers following means and mean differences represent 95\% confidence intervals, and all reported post hoc tests utilize Tukey's correction for multiple comparisons. Data for the historical influence ratings are presented in Table 1. We replicated ingroup inflation; people attributed on average $11 \%$ more of US history to their home state than did participants from other states. An ingroup inflation index was calculated as the difference between each participant's home state rating and the mean rating for that state as provided by out-ofstate raters. The mean of these difference scores differed from 0 (which would have indicated no difference between in- and out-of-state raters), as indicated by a one-sample t-test against a criterion of $0, \mathrm{M}_{\text {diff }}=.11[.07, .14], t(287)=6.16, p<.001, d$ $=0.36$. Interestingly, there was a difference in ingroup inflation across task-order counterbalancing conditions. Ingroup inflation was greater when historical influence rating occurred prior to the fluency task, $\mathrm{M}=.14[.09, .19]$, than when it followed the fluency task, $\mathrm{M}=.07[.02, .12], \mathrm{M}_{\text {diff }}=.07$ $[.003, .14], t(286)=2.06, p=.04, d=0.24$. We will comment on this intriguing finding, which is somewhat at odds with previous literature, in the Discussion.

\section{Parochial knowledge bias}

We next examined whether participants could more fluently think of historical events that had happened in their home state than could people from other states, that is, whether they showed a parochial knowledge bias. For each of the three states, participants had listed as many important historical events that occurred in that state as they could think of within $1 \mathrm{~min}$. Two human coders each independently scored the full set of these protocols, counting the number of unique events participants provided for each state. Events were coded as discrete events if they were recognizably different terms separated by a comma, as described in the instructions. While in the vast majority of cases this rule produced clear event counts, ambiguous choices were resolved by discussion, and the counts used for final analyses represent the mean of the 
Table 1 Experiment 1: Proportion of US history accounted for by each state, as judged by in- and out-of-state raters, with ingroup inflation as the difference between in- and out-of-state raters. P-values and Cohen's $d$ are provided for the ingroup inflation effect*

\begin{tabular}{|c|c|c|c|c|c|}
\hline State & Baseline (out-of-state raters) & In-state raters & Ingroup inflation & $p$ & $d$ \\
\hline Virginia & $.40[.36, .45]$ & $.54[.48, .60]$ & $.14[.08, .20]$ & $<.001$ & 0.48 \\
\hline Massachussetts & $.43[.39, .47]$ & $.52[.46, .58]$ & $.09[.01, .16]$ & .02 & 0.29 \\
\hline California & $.25[.21, .28]$ & $.34[.28, .39]$ & $.09[.03, .15]$ & .003 & 0.37 \\
\hline Mean & $.36[.33, .39]$ & $.46[.43, .50]$ & $.10[.07, .14]$ & $<.001$ & 0.37 \\
\hline
\end{tabular}

*Numbers in brackets represent $95 \%$ confidence intervals

two raters' scores for each cell. Coders were blind to participants' home state, counterbalancing condition, and all demographic information. There was strong agreement between the two coders, Cohen's $K=.89, p<.001$. Data for this fluency measure are provided in Table 2, along with the index of parochial knowledge bias. We calculated a parochial knowledge bias index as the difference between the number of events participants listed for their home state and the mean number of events out-of-state participants listed for that state.

Participants showed a parochial knowledge bias, as indicated by a one-sample t-test against a criterion of $0, \mathrm{M}_{\text {diff }}=$ $0.83[0.63,1.02], t(287)=8.34, p<.001, d=0.49$. For each state, in-state participants could more fluently list important historical events than could out-of-state participants. Retrieval of collective memories asymmetrically favored the ingroup.

Finally, we had predicted that the extent of the asymmetry in accessibility would correlate positively with ingroup inflation. That is, the more collective memory demonstrated preferential accessibility for ingroup-relevant events relative to outgroup-relevant events, the more judgments of historical influence should show group-centric overclaiming. We recalculated ingroup inflation and parochial knowledge bias as within-individual differences in ratings and accessibility. Whereas prior reports of ingroup inflation have calculated the index as the difference between in-state raters and an out-of-state baseline for each state (e.g., Churchill et al., 2019; Putnam et al., 2018), we were interested in differences of relative accessibility within the minds of individuals, and how such relative biases in accessibility influence judgments of historical influence. We thus calculated within-individual measures of ingroup inflation as the difference between the historical influence rating given to the home state and the mean of the ratings given to the two outgroup states by each participant. Likewise, a within-individual measure of parochial knowledge bias was calculated as the number of events a participant could list for their home state minus the mean number of events they could list for the other two states. Using these two within-individual measures, we again found both ingroup inflation, $t(287)=6.32, p<.001, d=0.37$, and a parochial knowledge bias, $t(287)=9.04, p<.001, d=0.53$ (see Fig. 1).

Crucial to our hypothesis, the more accessibility asymmetrically favored the home state, the more participants tended to inflate judgments of the home state's historical influence. That is, the parochial knowledge bias positively predicted ingroup inflation, $\mathrm{b}=5.59$ [3.66, 7.51], $p<.001$ (see Fig. 2). The model demonstrated good fit, $\mathrm{R}^{2}=.10, F(1,285)=32.71, p$ $<.001$.

\section{Experiment 1 Summary}

Participants demonstrated both ingroup inflation by rating their home-state as having been more influential than outside raters granted and a parochial knowledge bias, in that they could more fluently think of important historical events that had occurred in their home-state than outsiders were able to do. Importantly, the size of these relative asymmetries in memory and judgment were positively correlated. Having a relatively strong parochial bias in the collective memories that were easily accessible was

Table 2 Experiment 1: Mean number of important historical events in- and out-of-state participants listed for each state, with a measure of parochial knowledge bias as the difference between the two measures. P-values and Cohen's d are provided for the parochial knowledge bias effect*

\begin{tabular}{llllll}
\hline State & Baseline (out-of-state participants) & In-state participants & Parochial knowledge bias & $p$ & $d$ \\
\hline Virginia & $2.27[2.08,2.46]$ & $3.05[2.73,3.37]$ & $0.79[0.47,1.11]$ & $<.001$ & 0.55 \\
Massachussetts & $2.45[2.26,2.64]$ & $3.90[3.53,4.27]$ & $1.45[1.08,1.82]$ & 0.001 & 0.97 \\
California & $2.54[2.33,2.76]$ & $2.81[2.52,3.11]$ & $0.27[-0.02,0.57]$ & 0.15 & 0.18 \\
Mean & $2.42[2.28,2.56]$ & $3.26[3.06,3.45]$ & $0.83[0.64,1.03]$ & $<.001$ & 0.53 \\
\hline
\end{tabular}

*Numbers in brackets represent $95 \%$ confidence intervals 


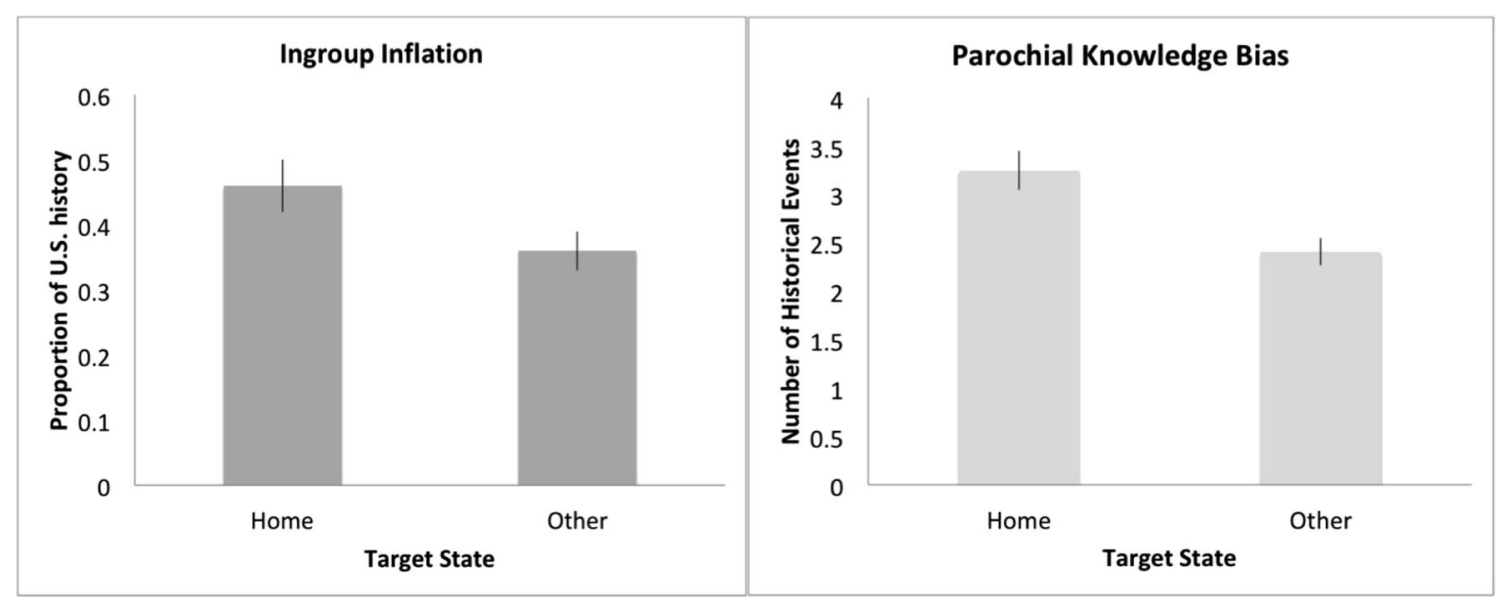

Fig. 1 Experiment 1: Participants more fluently listed historical events from their home states than from other states, and attributed more historical influence to their home state than other states. Error bars represent $95 \%$ confidence intervals

associated with inflated ratings of historical influence, and vice versa. Experiment 1 demonstrated the hypothesized relation between these two constructs. In Experiment 2, we tested the hypothesized causal relation by manipulating the relative accessibility of outgroup-relevant events in order to examine whether decreasing the asymmetry in availability would decrease inflated judgments of influence.

\section{Experiment 2}

If the availability heuristic explanation of ingroup inflation is correct, equating the relative accessibility of inand outgroup-relevant events should reduce overclaiming. In Experiment 2, participants engaged in the same retrieval fluency and historical influence-rating tasks as in

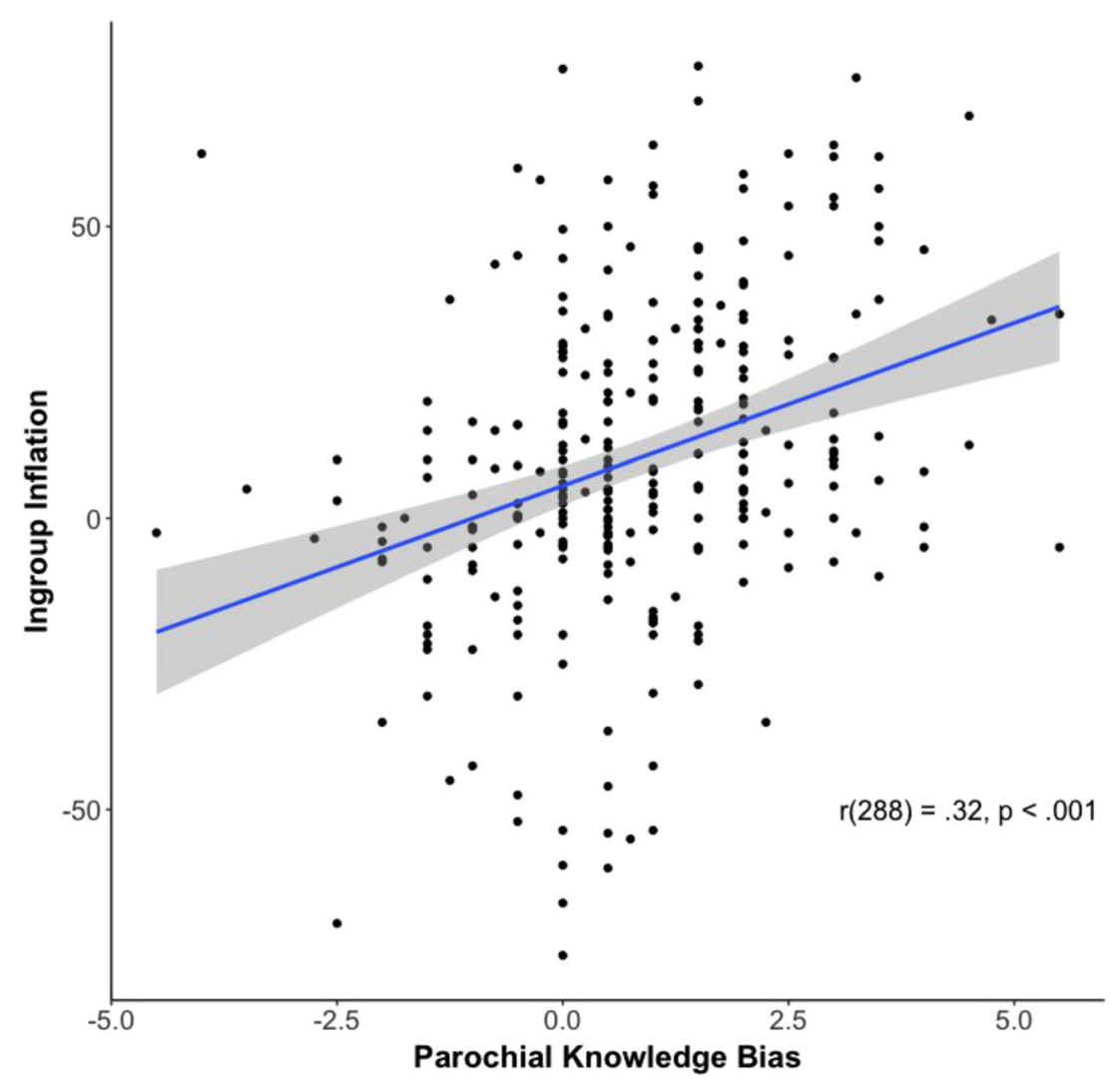

Fig. 2 Experiment 1: Having more ready access to events in the ingroup's history relative to the outgroup's (parochial knowledge bias) predicted a greater tendency to attribute more historical influence to the ingroup than to outgroups (ingroup inflation). Shading around the regression line represents $95 \%$ confidence intervals of the regression coefficient 
Experiment 1, with the alteration that participants retrieved only outgroup-relevant historical events during the retrieval fluency task. Targeted retrieval of outgrouprelevant events should leave the default accessibility of ingroup-related events relatively unaffected and could even decrease their relative accessibility via suppression of response competition (e.g., Stone et al., 2012; Storm \& Levy, 2012). It was predicted that this targeted retrieval would lead to a decrease in ingroup inflation.

\section{Methods}

\section{Participants}

Two hundred and seventy-three MTurk participants were recruited from the same target states as in Experiment 1, with the same inclusion criteria. People who had participated in Experiment 1 were blocked based on MTurk ID. Fifteen cases were excluded for providing nonsense responses (excluded $\mathrm{N}_{C A}=8 ; \mathrm{N}_{M A}=4 ; \mathrm{N}_{V A}=3$ ), for a final sample of 257. The final sample sizes for California ( $\mathrm{n}=$ 92), Massachusetts $(n=84)$, and Virginia $(n=82)$ were satisfactory given an a priori power analysis for replicating the within-individual ingroup inflation effect size from Experiment $1(d=0.37)$, which required a minimal sample size of 47 . The $\mathrm{G}^{*}$ Power protocol is provided in the OSM: Appendix I.

The average age of participants was 37 years, ranging from 18 to 76 years. Forty percent of the sample was female, with the remainder male except for one person who reported gender nonconforming. Again, the plurality of participants had a fouryear degree (48\%), with $18 \%$ having a professional degree, $16 \%$ some college (but no degree), $4 \%$ having a high school degree, $11 \%$ having a two-year degree, and $3 \%$ having a doctorate. Mean endorsement of the statement "the state I grew up in is an important part of my personal identity" was 2.28 [2.27, 2.29], between "a little" and "a moderate amount." Participants from different states did not differ in the degree of identification with their home state, $p>.05$.

\section{Procedures}

Participants performed the same two tasks as in Experiment 1: historical influence ratings for the three target states, and a timed fluency task. Counterbalancing of task order and item order within task remained the same. The one procedural difference was that in the timed fluency task, participants only retrieved events for the two outgroup states, selectively targeting outgroup-relevant events and leaving ingrouprelevant events unmanipulated. This allowed a test of the primary hypothesis of Experiment 2, which was that increasing the accessibility of outgroup-relevant events relative to ingroup-relevant events should decrease ingroup inflation.

\section{Results}

Historical influence rating data for each state are presented in Table 3. Ingroup inflation as a within-subjects effect replicated, though with a smaller effect, with participants again tending to attribute more of US history to their home state $(\mathrm{M}=.34, \mathrm{SE}=1.45)$ than they did to other states $(\mathrm{M}=.28$, $\mathrm{SE}=1.26), \mathrm{M}_{\text {diff }}=.06[.03, .08], t(257)=4.67, p<.001, d=$ 0.29 . Unlike Experiment 1, the interaction between task order and in/outgroup target did not attain statistical significance, $F(1,256)=3.46, p=.06$.

In order to address the possibility that the interaction was insufficiently powered, we combined datasets from Experiments 1 and 2. On this combined dataset $(\mathrm{N}=546)$, we again tested for (a) ingroup inflation, (b) whether this effect differed between Study 1 and Study 2, with the prediction that the Study 2 manipulation would have reduced ingroup inflation relative to the Study 1 manipulation, and (c) the expected effect of task counterbalancing order, with participants who had taken the fluency task first showing reduced ingroup inflation relative to those who had taken the historical influencerating task first. To that end, we conducted a 2 (Group Referent: ingroup, outgroup) $\times 2$ (Experiment Number: 1,2) $\times 2$ (Task Counterbalancing Condition: Rating First, Fluency Task First) mixed ANOVA.

There was the main effect of group referent, $F(1,542)=$ $58.30, p<.001$, in which greater influence was attributed to the ingroup than to the outgroup, $\mathrm{M}_{\text {diff }}=.08[.06, .10], t(545)$ $=7.64, p<.001, d=0.34$. Of primary interest given the hypothesis of Experiment 2, there was also an interaction between group referent and experiment number, $F(1,542)=$ $4.82, p=.03$. Ingroup inflation was smaller in Experiment 2 where the fluency task targeted only outgroup-relevant events, Inflation Index $=.06[.03, .08], t(257)=4.67, p<.001, d=$ 0.29 , than when participants had retrieved both outgroup- and ingroup-relevant events, Inflation Index $=.10[.07, .13]$, $t(287)=6.32, p<.001, d=0.37$. There was a main effect of experiment, with participants providing overall lower ratings in Experiment $2(\mathrm{M}=.32[.29, .34])$ than in Experiment $1(\mathrm{M}$ $=.42[.39, .44]), F(1,542)=27.38, p<.001$. No other main effect or interaction attained statistical significance, all $p>.05$.

Identification with the home state correlated positively with the ingroup inflation index, $r(544)=.23, p<.001$. Tellingly, this correlation was somewhat stronger than the correlation between state identification and the ingroup rating alone, $r(544)=.19, p$ $<.001$. Because the Experiment 2 manipulation excluded selective retrieval of ingroup relevant events, changes in parochial knowledge bias could not be measured directly in this design.

\section{Experiment 2 Summary}

Experiment 2 provided converging evidence that ingroup inflation arises in part because when people make judgments 
Table 3 Experiment 2: Proportion of US history accounted for by each state, as judged by in- and out-of-state raters, with ingroup inflation as the difference between the two measures. P-values and Cohen's $d$ are provided for the ingroup inflation effect*

\begin{tabular}{|c|c|c|c|c|c|}
\hline State & Baseline (out-of-state raters) & In-state raters & Ingroup inflation & $\mathrm{p}$ & $\mathrm{d}$ \\
\hline Virginia & $.32[.31, .321]$ & $.37[.37, .38]$ & $.05[-.001, .11]$ & .08 & 0.24 \\
\hline Massachussetts & $.31[.31, .313]$ & $.41[.41, .42]$ & $.10[.04, .16]$ & .001 & 0.43 \\
\hline California & $.22[.215, .22]$ & $.25[.25, .26]$ & $.04[-.01, .09]$ & .15 & 0.19 \\
\hline Mean & $.28[.28, .29]$ & $.34[.34, .343]$ & $.06[.03, .08]$ & $<.001$ & 0.29 \\
\hline
\end{tabular}

*Numbers in brackets represent $95 \%$ confidence intervals

about historical influence, those judgments are informed by a confluence of the availability heuristic and biased accessibility to collective memories. Experiment 2 targeted retrieval of outgroup-relevant historical events, under the assumption that this would decrease the relative asymmetry between the availability of in- and outgroup-relevant historical events, i.e., would decrease the parochial knowledge bias demonstrated in Experiment 1. Relative to Experiment 1, participants in Experiment 2 did indeed show decreased ingroup inflation.

\section{Experiment 3}

In the final Experiment of the study, we sought to extend evidence for the relation between ingroup inflation and the parochial knowledge bias using a multiple-item rather than a single-item measure of ingroup inflation. In the extant literature, the ingroup inflation index derives from a one-item measure: the difference between in- and out-of-state ratings for a particular state (or country). In Experiment 3, we developed a multi-item test for measuring ingroup inflation, with the dual goals of conceptual replication using a new tool and of developing a more stable metric.

\section{Methods}

\section{Materials}

We developed for each of the three target states (California, Massachusetts, and Virginia) a set of 30 multiple choice questions, each targeting an important historical event that had occurred in that state, for a total of 90 questions. The full set of questions is provided in Appendix III of the OSM. These events were drawn from the respective states' Wikipedia pages and other online sources of state history. Each question had three lures and one correct answer. Questions appeared one per page in a Qualtrics survey. Beneath each question was a 5-point scale where participants rated how confident they were that their choice was correct. On the next page, participants saw the correct answer, and rated how important that event was to the history of the USA on another 5-point scale. This task was self-paced.

\section{Participants}

Three hundred and two participants were recruited from the same three target states using the same methods and criteria as in the prior studies. MTurk IDs from participants who had participated in prior studies were blocked. Their mean age was 36 years, with a range from 20 to 79 years. Forty-eight percent of the sample identified as female. Again, the plurality of participants had college degrees at $46 \%, 18 \%$ had some college but no degree, $15 \%$ had a professional degree, $10 \%$ had an associates degree, $7 \%$ had a high school degree, 3\% had a doctorate, and $.007 \%$ had less than a high school degree. Participants reported that their home state represented an important part of their personal identity, $\mathrm{M}=3.43,95 \%$ CI [3.30, 3.56], between "A lot" and "A great deal." Participants from different states did not differ in the extent to which their home state was important to their personal identity, $p>.05$.

\section{Procedures}

Participants followed a link from MTurk to a Qualtrics survey (Appendix IV in the OSM). At the survey, they were instructed that they would see a set of questions probing historical knowledge about US state history. They were enjoined not to look up any answers, as we were simply interested in what people knew. At the end of the survey we asked participants whether they had looked up any answers, and those who indicated "Yes" $(n=4)$ were removed from the sample.

Each of the three states had a bank of 30 questions about important historical events that had occurred in that state. For each participant, ten of these questions from each state were randomly selected, so that each participant saw 30 multiplechoice questions total. Question presentation was blocked by state. The order of state presentation was randomized for each participant, and within each state the order of the ten questions was randomized. The order of the four multiple-choice options was also randomized for each question and for each participant, such that the correct answer had an equal likelihood of appearing in any position. Each question appeared individually on its own page, with the confidence rating scale below the question. After answering each question and providing a confidence rating, participants advanced to the next 
page, where they saw the correct answer, and rated the importance of that event to US history. After answering each of the 30 questions, providing a confidence rating for each choice, and rating the importance of each event, participants provided the same demographic information as previous studies. Participants were then reimbursed US\$1.

\section{Results}

\section{Parochial knowledge bias}

Data for in- and out-of-state accuracy and confidence ratings are presented in the first two rows of Table 4. Participants responded correctly to questions concerning events that had happened in their home state more frequently than events that had happened in other states, $t(301)=8.07, p<.001, d=0.11$. They were also more confident in their choices when they responded to in- versus out-of-state questions, $t(301)=9.90$, $p<.001, d=0.57$. Confidence correlated modestly with accuracy, $r(300)=.26, p<.001$. Thus, both subjective metacognitive judgments and actual accuracy asymmetrically favored the home state versus other states, providing converging evidence for a parochial knowledge bias.

\section{Ingroup inflation}

We calculated within-individual measures of ingroup inflation using methods similar to those described in the previous experiments. For each individual, we calculated the mean importance ratings for events that occurred in the home state and the mean of importance ratings for the other two states. The difference between these two sets of importance ratings represented the ingroup inflation index. Data are presented in the third row of Table 3. Using this method, ingroup inflation was not statistically significant, but was numerically in the right direction, $t(301)=1.96, p=.05, d=0.11$.

Table 4 Experiment 3: Mean accuracy, confidence that choice was correct, and historical importance rating for multiple choice items targeting historical events from the home state and other states. Inflation Index represents a measure of the parochial knowledge bias and ingroup inflation*

\begin{tabular}{llll}
\hline Measure & \multicolumn{2}{l}{ Group referent } & \multirow{2}{*}{ Inflation index } \\
\cline { 2 - 3 } & Home state & Other state & \\
\hline Accuracy & $.51[.49, .53]$ & $.43[.41, .45]$ & $.08[.06, .10]$ \\
Confidence & $2.78[2.69,2.87]$ & $2.48[2.40,2.56]$ & $0.29[0.24,0.35]$ \\
Importance & $3.32[3.23,3.41]$ & $3.25[3.18,3.32]$ & $0.07[0.00,0.13]$ \\
\hline
\end{tabular}

*Numbers in brackets represent $95 \%$ confidence intervals

\section{Relation between ingroup inflation and parochial knowledge bias}

A greater asymmetry in knowledge correlated positively with a greater asymmetry in confidence, $r(300)=.23, p<.001$. However, a greater asymmetry in accuracy did not correlate significantly with a greater asymmetry in ratings of importance, $r(300)=.02, p=.77$.

\section{Experiment 3 Summary}

In Experiment 3 we targeted knowledge and ratings of importance for specific events that had occurred within each of the three target states. Participants answered multiple-choice questions about these events, rated confidence in their answers, and rated the historical importance of each event. We conceptually replicated the parochial knowledge bias using this multiple-item measure, in that participants were both more accurate and more confident on items concerning events that had occurred in their homestate versus other states. However, we did not find ingroup inflation using ratings of historical importance attached to specific, concrete events, and there was no correlation between the multiple-item measures of parochial knowledge bias and ingroup inflation.

\section{Discussion}

We had hypothesized that people overclaim historical influence for their ingroups in part because they make such judgments using the availability heuristic and have asymmetrical accessibility to ingroup- and outgroup-relevant historical events. We replicated the basic phenomenon of ingroup inflation and demonstrated for the first time that ingroup-relevant historical events are cognitively more available than outgrouprelevant ones - thus that participants showed a parochial knowledge bias. Participants could, on average, retrieve important historical events more fluently when those events had occurred within their home state. We further showed that historical over-claiming is positively related to the gradient of this asymmetrical accessibility. Prior work has conjectured this relation, but this is the first time it has been demonstrated empirically using real-world collective memories.

The effect of task order on ingroup inflation in Experiment 1 bears comment. Participants who had just spent 3 min thinking of important historical events in their home state and two other states tended to show lower levels of ingroup inflation than participants who made their influence judgments prior to the fluency task. This suggests that a robust parochial knowledge bias is in some sense the default, but that explicitly retrieving other groups' historical contributions from memory could 
reduce the asymmetry, and subsequently reduce ingroup inflation. This pattern is somewhat curious given that Putnam et al. (2018), who administered a historical questionnaire either before or after participants made their historical influence judgments, found no effect of the questionnaire's placement on ingroup inflation. The difference might be due to the fact that our fluency probe elicited very targeted retrieval of events from the outgroup states, whereas Putnam et al.'s (2018) questionnaire was relatively general. That is, their questions did not necessarily target events specifically from the home state or the particular non-resident states participants rated and thus did not necessarily cue retrieval of events unique to the particular outgroups against which people were making their judgments. The test for differences in ingroup inflation across task presentation order, however, was very close to the threshold of significance $(p=.04)$, so Experiment 2 attempted to address the question more directly.

Experiment 2 targeted retrieval of outgroup relevant events to the exclusion of ingroup relevant events. The interaction between task order and group referent remained non-significant $(p=.06)$, even after combining datasets from Experiments 1 and 2, although ingroup inflation was indeed reduced in Experiment 2 as predicted. We should issue the caveat for this analysis on the combined dataset that, although random assignment happened within each experiment, for the combined analysis participants from a single sample were not randomly assigned to the conditions analyzed. However, because the Experiment 1 and 2 samples were collected within a relatively short time of one another using the same pool and inclusion/exclusion criteria, there is no reason to believe that the two samples differed systematically. The ambiguity surrounding the interaction or lack thereof between counterbalancing order and ingroup inflation merits investigation in future work. These interactions may be inconclusive because the retrieval fluency task could have exerted one of two antagonistic influences on the judgments underlying ingroup inflation. On the one hand, as intended, retrieving outgrouprelevant events could have increased their relative availability to the subsequent judgments of historical influence, decreasing ingroup inflation. On the other hand, if people attempted to retrieve outgroup-relevant events and found the task unexpectedly difficult, metacognitive reasoning may have led them to conclude that if it were so difficult to think of events for the outgroups, those groups must not have accomplished much. Such metacognitive evaluations deriving from ease (or difficulty) of retrieval have been demonstrated both for controlled experimental materials and collective memories (e.g., Echterhoff \& Hirst, 2006;
Schwartz et al. 1991). If these two possible consequences of targeted retrieval of outgroup-relevant events occurred for different participants, the net impact of retrieving outgroup-relevant events on ingroup inflation would have been muddied. Future work should investigate more systematically the conditions under which targeted retrieval decreases ingroup inflation by increasing the availability of outgroup-relevant events, versus when it increases ingroup inflation because of metacognitive appraisals of difficulty. Despite this lingering ambiguity, the main effect of ingroup inflation, measured as a within-subject effect, did decrease as predicted. This suggests that although ingroup inflation may arise from default biases in collective memory, it may be sensitive to targeted cognitive interventions.

Experiment 3 indicated that although the parochial knowledge bias replicated using a multi-item recognition measure of accuracy rather than fluency during free recall, the effect was smaller, with parochial knowledge bias $d=0.55$ in the retrieval fluency measure versus $d=0.11$ in the multiple-choice recognition measure. Further, the index of ingroup inflation derived from a multiple-item metric based on importance of specific events was not statistically significant, and did not correlate with the parochial knowledge bias derived from accuracy on multiple-choice items. There are several conclusions we might draw from these findings. First, in our materials, the group relevance of particular events may not have always been strongly salient. Individual questions were not marked explicitly as having occurred in a particular state, so their relation to the target states may have been less obvious than in the fluency task, where the target state was essentially the cue triggering retrieval. This weaker connection with the relevant group identity may partially explain why ingroup inflation was marginal in the individual item importance ratings. Future work could manipulate how saliently particular events are linked with group identity to see whether this is the case.

Second, and in line with our primary hypothesis, it seems likely that it is ease of access per se, rather than more stable knowledge or judgments regarding specific concrete events, that informs the pattern of judgments underlying ingroup inflation. Ingroup inflation seems primarily to arise because of a relatively small number of events that come to mind easily, rather than any deeper reflection, and so is sensitive to cognitive manipulations that either increase the accessibility of outgroup-relevant events (Experiment 2) or require relatively concrete evaluation of specific events (Experiment 3). This relative importance of fluid accessibility may be a function of the particular social entities we used in this study. For Americans enculturated in the American 
milieu of memory, asymmetrical availability of historical information between different US states may be relatively moderate. The situation could have been different if we had compared asymmetries in availability for, say, historical events from America's past versus Russia's or Vietnam's. American states were selected for the current study in part because of methodological tractability, but other collective identities could reveal themselves to be situations where asymmetries in availability are more influential.

\section{Conclusion}

Overclaiming of historical influence can in part be attributed to the application of the availability heuristic to a biased store of collective memories. This bias in the context of US states seems to be primarily an ingroup-centered bias in accessibility, although the cognitive characteristics of the parochial knowledge bias may differ in reference to other types of social identities. Manipulations that decrease the accessibility bias in collective memory may support judgments less characterized by chauvinistic overclaiming. This work builds on an accumulating body of research into the cognitive characteristics of collective memories and social representations of history.

\section{References}

Churchill, L., Yamashiro, J., and Roediger, H.L. III. (2019). Moralized memory: Binding values predict inflated estimates of the group's historical influence. Memory, 27(8), 1099-1109. DOI: https://doi. org/10.1080/09658211.2019.1623261

Echterhoff, G., and Hirst, W. (2006). Thinking about memory for everyday and shocking events: Do people use ease of retrieval cues in memory judgments? Memory \& Cognition, 34, 763-775. doi: https://doi.org/10.3758/bf03193424.

Graham, J., Haidt, J., and Nosek, B. A. (2009). Liberals and conservatives rely on different sets of moral foundations. Journal of Personality and Social Psychology, 96(5), 1029-1046. https://doi.org/10.1037/ a0015141

Hirst, W. and Manier, D. (2008). Towards a psychology of collective memory. Memory, 16(3), 183-200. https://doi.org/10.1080/ 09658210701811912

Hirst, W., Yamashiro, J. K., and Coman, A. (2018). Collective Memory from a Psychological Perspective. Trends in Cognitive Science, 22, 438-451. DOI: https://doi.org/10.1016/j.tics.2018.02.010

Lichtenstein, S., Slovic, P., Fischhoff, B., Layman, M., and Coombs, B. (1978). Judged frequency of lethal events. Journal of Experimental Psychology: Human Learning and Memory, 4, 551-578. https://doi. org/10.1037/0278-7393.4.6.551

MacLeod, A.K. and Byrne, A. (1996). Anxiety, Depression, and the anticipation of future positive and negative experiences. Journal of
Abnormal Psychology, 105, 286-289. https://doi.org/10.1037/0021843X.105.2.286

MacLeod, A. K., Rose, G. S., \& Williams, J. M. G. (1993). Components of hopelessness about the future in parasuicide. Cognitive Therapy and Research, 17, 441-455. https://doi.org/10.1007/BF01173056

MacLeod, A. K., Tata, P., Kentish, J., \& Jacobsen, H. (1997). Retrospective and prospective cognitions in anxiety and depression. Cognition and Emotion, 11, 467-479. https://doi.org/10.1080/ 026999397379881

Nguyen, V.T. (2016). Nothing Ever Dies: Vietnam and the Memory of War. Cambridge, MA: Harvard University Press.

Novick, P. (1999). The Holocaust in American Life. Boston: Houghton Mifflin.

Putnam, A.L., Ross, M.Q., Soter, L.K., and Roediger, H.L., III. (2018). Collective Narcissism: Americans exaggerate the role of their home state in appraising U.S. history. Psychological Science, 29(9), 14141422. doi: https://doi.org/10.1177/0956797618772504

Roediger, H.L., III and Abel, M. (2015). Collective memory: a new arena of cognitive study. Trends in Cognitive Science, 19(7), 359-361. https://doi.org/10.1016/j.tics.2015.04.003

Roediger, H.L., III, Abel, M., Umanath, S., Shaffer, R.A., Fairfield, B., Takahashi, M., and Wertsch, J.V. (2019). Competing national memories of World War II. Proceedings of the National Academy of Sciences, 116(34), 16678-16686. https://doi.org/10.1073/pnas. 1907992116

Roediger, H.L., III, Putnam, A., and Yamashiro, J. (2020). National and state narcissism as reflected in overclaiming. In J. Wertsch \& H.L. Roediger, III (Eds.), National Memory in a Time of Populism. New York, NY: Oxford University Press. In Press.

Ross, M. and Sicoly, F. (1979). Egocentric biases in availability and attribution. Journal of Personality and Social Psychology, 37(3), 322-336. https://doi.org/10.1037/0022-3514.37.3.322

Ross, M.Q., Sterling-Maisel, O.A., Tracy, O., and Putnam, A.L. (2020). Overclaiming responsibility in ficitious countries: Unpacking the role of availability in support theory predictions of overclaiming. Memory \& Cognition, in press.

Schwarz, N., Bless, H., Strack, F., Klumpp, G., Rittenauerschatka, H., and Simons, A. (1991). Ease of retrieval as information - Another look at the availability heuristic. Journal of Personality and Social Psychology, 61(2), 195-202. DOI: https://doi.org/10.1037//00223514.61.2.195

Shrikanth, S., Szpunar, P. M., \& Szpunar, K. K. (2018). Staying Positive in a Dystopian Future: A Novel Dissociation Between Personal and Collective Cognition. Journal of Experimental Psychology: General, 147(8), 1200-1210. https://doi.org/10.1037/xge0000421

Stone, C., Coman, A., Brown, A.D., Koppel, J., and Hirst, W. (2012). Toward a science of silence: The consequences of leaving a memory unsaid. Perspectives on Psychological Science, 7(1), 39-53. https:// doi.org/10.1177/1745691611427303

Storm, B., and Levy, B. (2012). A progress report on the inhibition account of retrieval induced forgetting. Memory \& Cognition, 40(6), 827-843. doi: https://doi.org/10.3758/s13421-012-0211-7.

Tulving, E. and Pearlstone, Z. (1966). Availability versus accessibility of information in memory for words. Journal of Verbal Learning \& Verbal Behavior, 5(4), 381-391. https://doi.org/10.1016/S00225371(66)80048-8

Tversky, A. and Kahneman, D. (1973). Availability: A heuristic for judging frequency and probability. Cognitive Psychology, 5, 207-232. https://doi.org/10.1016/0010-0285(73)90033-9

Wertsch, J. (2002). Voices of Collective Remembering (1st ed.). New York, NY: Cambridge University Press. 
Wertsch, J., \& Roediger, H. I. (2008). Collective Memory: Conceptual Foundations and Theoretical Approaches. Memory, 16(3), 318-326. https://doi.org/10.1080/09658210701801434

Zaromb, F. M., Liu, J., Hanke, K., Putnam, A. L., \& Roediger, H. L. (2018). We Made History: Citizens of 35 countries overestimate their nation's role in world history. Journal for Applied Research in Memory and Cognition, 7(4), 521-528. https://doi.org/10.1016/j. jarmac.2018.05.006

Open Practices Statement The datasets, Qualtrics surveys, and all online supplemental material referenced in the article are available on the Open Science Framework: "Ingroup Inflation and Availability," https://osf.io/ etxgr/. Studies 1 and 3 were pre-registered at https://osf.io/z4xvc.
Author Note This project was supported by a research fund from the Princeton School for Public and International Affairs to J.K.Y and a grant from the James S. McDonnell Foundation to H.L.R. We would like to thank Adam Putnam for early feedback, as well as Jesse Rubin and Medhavi Verma for their research assistance. J.K.Y. is currently affiliated with the department of psychology at the University of California, Santa Cruz. We report no conflicts of interest.

Publisher's note Springer Nature remains neutral with regard to jurisdictional claims in published maps and institutional affiliations. 